Orange Journal / Volumen 2 Número 4/ Julio - diciembre 2020

DOI: https://doi.org/10.46502/issn.2710-995X/2020.4.03

\title{
Uso de fármacos en el anciano, un desafío a enfrentar. Reporte de un caso
}

\author{
Drug use in the elderly, a challenge to confront. Report of a case \\ Recibido: 28 de diciembre de 2020 \\ Aceptado: 10 de mayo de 2021 \\ Escrito por: \\ Irma Leonor Ortega López ${ }^{7}$ \\ https://orcid.org/0000-0001-7174-1808 \\ Natacha de la Concepción Sagaró Yi ${ }^{8}$ \\ https://orcid.org/0000-0002-6262-5757
}

\section{Resumen}

Los adultos mayores son especialmente vulnerables a los efectos adversos de medicamentos por lo que es necesario realizar intervenciones para su reducción. Es un hecho que los mejores resultados en salud se obtienen cuando los servicios de salud están organizados en torno a las necesidades y expectativas de salud de las personas, no en torno a las enfermedades. En este sentido se presenta un caso y la estrategia de seguimiento para minimizar la incidencia de los problemas relacionados con medicamentos en un paciente con comorbilidades y polifarmacia.

Palabras clave: Anciano. Medicación potencialmente inapropiada. Problemas relacionados con los medicamentos. Reacción adversa a medicamento. Seguimiento farmacoterapéutico.

\begin{abstract}
The elderly are specially vulnerable to the adverse effects of medications for what is necessary to carry out interventions for their reduction. It is a fact that the best results in health are obtained when the services of health are organized around the necessities and expectations of people's health, not around the illnesses. In this sense it is presented a case and the pursuit strategy to minimize the incidence of the problems related with medications in a patient with comorbidities and polypharmacy.
\end{abstract}

Key Words: Drug adverse reaction, Drugs related problems, Elderly, Pharmacotherapy follow- up, Potentially inappropriate medications.

\footnotetext{
${ }^{7}$ Dr.C., Profesor Titular. Departamento de Farmacia. Universidad de Oriente. Santiago de Cuba. Cuba.

8 Dra. en Ciencias Médicas. Especialidad de 1er grado en Geriatra. Hogar de Ancianos "América Labadí Arce". Santiago de Cuba, Cuba.
} 


\section{Introducción}

Los adultos mayores (AM) son especialmente vulnerables a los efectos adversos de medicamentos por muchas razones, entre las que destacan los cambios farmacocinéticos y farmacodinámicos relacionados con el envejecimiento, la multimorbilidad, la polifarmacia y, la escasa formación de los profesionales en farmacología geriátrica.

Las consecuencias de esto son un aumento del número y la gravedad de las reacciones adversas a medicamentos (RAM), que empeora su salud y su calidad de vida, aumentando en consecuencia el uso de recursos sanitarios y los costos de la atención (Cruz, 2017); de aquí la necesidad de realizar intervenciones con vista a su reducción (Lavan, Gallagher, Parsons, y O’Mahony, 2017). Lavan, Gallagher, Parsons, y O’Mahony, (2017).

La situación se torna más compleja ya que la población suele ser atendida por diferentes médicos, en diferentes niveles de atención e incluso en diferentes instituciones de salud, lo que puede fragmentar el tratamiento al no existir sistemas que permitan contar con información completa y necesaria para la correcta prescripción (Poblano, Bautista, Acosta, Gómez y Saturno, 2020). Por ello es necesario que los servicios de salud estén organizados en torno a las necesidades y expectativas de salud de las personas, no en torno a las enfermedades (Organización Mundial de la Salud [OMS], 2015).

\section{Marco Teórico}

Polifarmacia: uso rutinario de cinco o más fármacos; aunque se propone la introducción paulatina de los términos "polifarmacia apropiada y polifarmacia inapropiada", para referirse a la prescripción racional e irracional de múltiples medicamentos, basada en la mejor evidencia disponible y considerando factores individuales del paciente y contexto (World Health Organization, 2019).

Deprescripción: es la retirada supervisada por un profesional médico de fármacos inapropiados (Krska, Howard y Barnet, 2017).

\section{Metodología}

Se realizó seguimiento farmacoterapéutico, según procedimiento normalizado de trabajo para el AM propuesto por Ortega, (2018), a una anciana con comorbilidades y polifarmacia.

\section{Presentación del caso}

Paciente ambulatorio, de sexo femenino, de 72 años, raza negra, $65 \mathrm{Kg}$ de peso, y talla $1.72 \mathrm{~m}$. Requiere ayuda técnica (bastón) para deambular, tiene buena relación social y es apoyada por la familia.

Antecedentes patológicos personales: hipertensión arterial ( \pm 10 años), asma bronquial (desde la niñez); glaucoma ( \pm cinco años) y artrosis generalizada ( \pm 20 años). 


\section{ORANGE JOURNAL}

Tratamiento farmacológico habitual: verapamilo $(80 \mathrm{mg}) \quad 1 / 2$ tableta/cada $12 \mathrm{~h}$; hidroclorotiazida $(25 \mathrm{mg})$ 1/2 tableta/9:00 am; dorzolamida (2\%) una gota en ambos ojos/dos veces al día; salbutamol spray $(100 \mu \mathrm{g})$ de tres-cuatro administraciones diarias, si síntomas; carbamazepina (200 mg) una tableta/día.

Motivo de consulta: estreñimiento, nauseas, anorexia, dolor de cabeza, fatiga y dolores musculares.

\section{Resultados y discusión}

\section{Evaluación inicial de la paciente}

Del análisis de los datos obtenidos tras la revisión de la historia clínica, evaluación geriátrica funcional, anamnesis farmacológica y complementarios realizados, se constata: valores bajos de sodio en sangre $(100 \mathrm{mEq} / \mathrm{L})$, cifras tensionales de 130/90, irritabilidad, espasmos musculares y confusión mental ocasional.

La paciente refiere el uso de: metocarbamol $500 \mathrm{mg}$ una tableta/9:00 pm y loratadina 10 mg una tableta al acostarse; por lo que consume un total de siete medicamentos.

Fueron identificados PRM o sospecha de ellos, asociados a la indicación y la seguridad de la medicación (Tabla 1).

Entre los PRM asociados a la indicación se detectó la automedicación y la omisión de la prescripción para un Problema de Salud (PS)

La paciente se automedicó con loratadina y metocarbamol, medicamentos que incrementan la carga anticolinérgica, teniendo en cuenta el resto de la medicación que consume (Rudolph, Salow, Angelini y McGlinchey, 2008). Además, la loratadina está contraindicada en el glaucoma de ángulo cerrado (Ministerio de Salud Pública [MINSAP], 2018).

Publicaciones recientes (American Geriatrics Society [AGS], 2019), refieren que los relajantes musculares (metocarbamol), son considerados potencialmente inapropiados para el AM, ya que son pobremente tolerados a causa de sus efectos anticolinérgicos, por lo que deben ser evitados pues los riesgos superan claramente los beneficios. Elementos estos importantes en esta paciente la cual presenta limitaciones del movimiento.

Se detectó que la paciente tenía no tiene tratamiento para la artrosis generalizada. 
Tabla 1.

Caracterización de los PRM en la paciente.

\begin{tabular}{|c|c|c|c|}
\hline $\begin{array}{c}\text { Categoría del } \\
\text { PRM }\end{array}$ & $\begin{array}{c}\text { Descripción del } \\
\text { PRM }\end{array}$ & Causa del PRM & Medicamentos involucrados \\
\hline $\begin{array}{c}\text { Medicación } \\
\text { innecesaria }\end{array}$ & $\begin{array}{c}\text { Aumento del } \\
\text { riesgo RAM }\end{array}$ & Automedicación & loratadina, metocarbamol \\
\hline $\begin{array}{c}\text { Necesidad de } \\
\text { Medicación } \\
\text { adicional. }\end{array}$ & $\begin{array}{c}\text { Artrosis } \\
\text { generalizada }\end{array}$ & $\begin{array}{c}\text { Problema de salud no } \\
\text { tratado }\end{array}$ & antiinflamatorio, antirreumatoideo \\
\hline RAM & Hiponatremia & $\begin{array}{c}\text { Interacción } \\
\text { medicamentosa }\end{array}$ & $\begin{array}{c}\text { carbamazepina, hidroclorotiazida, } \\
\text { loratadina y } \\
\text { verapamilo }\end{array}$ \\
\hline & Estreñimiento & & $\begin{array}{c}\text { verapamilo, carbamazepina, } \\
\text { loratadina y metocarbamol }\end{array}$ \\
\hline
\end{tabular}

Fuente: Elaborada por lo autores

Entre los problemas de seguridad estuvo el estreñimiento y la hiponatremia, ambos asociados a interacción medicamentosa que provoca efectos indeseables, por potenciación de efectos.

Según Eschenhagen (2019), el estreñimiento es un efecto secundario común del verapamilo. Se plantea que la carga anticolinérgica que se produce al utilizar dos o más sustancias con esta actividad, incrementa el riesgo de toxicidad antimuscarínica (Delgado, et al. 2015; AGS, 2019).

Los riesgos asociados al uso de carbamazepina en el AM son superiores a los beneficios que aporta su uso, por lo que debe ser utilizada con precaución. Este medicamento puede exacerbar o causar el síndrome de secreción inapropiada de la hormona antidiurética. Si es necesario su uso deben ser monitorizados los niveles de sodio periódicamente ( $A G S$, 2019). Sus concentraciones plasmáticas se elevan por el uso concomitante con hidroclorotiazida, loratadina y verapamilo, lo cual incrementa el riesgo de estos efectos adversos (MINSAP, 2018).

\section{Diseño e implementación de un plan terapéutico de cuidado al paciente}

Según Cahn et al. (2015), los objetivos del tratamiento deben individualizarse a cada paciente, teniendo en cuenta la esperanza de vida, la edad, las condiciones médicas, etc. Objetivos terapéuticos para esta paciente:

Niveles de tensión arterial 130 a 140/90 mmhg.

$\checkmark$ Reducir el dolor articular y espasmos musculares.

$\checkmark$ Incrementar el número de deposiciones a la semana (de una, a dos o tres).

$\checkmark$ Incrementar los niveles de sodio sanguíneo a valores entre 130 y $145 \mathrm{mEq} / \mathrm{L}$. 


\section{ORANGE JOURNAL}

Propuestas de intervenciones farmacéuticas:

a) Educación sanitaria a la paciente: riesgo de la automedicación e importancia de los hábitos de vida saludables para reducir el estreñimiento y mejorar la artrosis.

b) Deprescripción del verapamilo.

El verapamilo tiene más efectos secundarios cardiacos y un alto potencial de interacción con otros medicamentos, y por tanto, no se considera como antihipertensivo de primera línea. Una alternativa de tratamiento, en pacientes de raza negra, pudiera ser el amlodipino, cuyo efecto no deseado más frecuente es el edema periférico; efecto secundario inofensivo, aunque posiblemente perturbador (Eschenhagen, 2019), por lo que habría que valorar las preferencias del paciente.

Smith, Cameron, Metcalf, Karen y Wilcox (2019), plantean que los inductores de CYP3A4 como la carbamazepina, reducen la biodisponibilidad del verapamilo, de igual manera este medicamento es un fuerte inhibidor de CYP3A4 y causa interacciones farmacológicas clínicamente relevantes con otros sustratos de CYP3A4. Este alto potencial del verapamilo para las interacciones entre los medicamentos es una clara desventaja y una de las razones de la disminución de su uso.

c) Iniciar tratamiento para reducir dolor articular.

Delgado et al. (2015), recomiendan el uso de paracetamol, asociado con ibuprofeno si fuera necesario, este último no más de tres meses dado el riesgo incrementado de sangrado en uso prolongado.

d) Deprescripción del metocarbamol y loratadina; limitar el uso del antihistamínico a situaciones de crisis de la enfermedad respiratoria.

La práctica de la deprescripción está aumentando como respuesta a la polimedicación (Krska, et al. 2017). La prescripción de varios fármacos debe adecuarse a las condiciones del paciente, teniendo en cuenta los riesgos de efectos adversos e interacciones de los fármacos implicados (Escuela Andaluza de Salud Pública, 2017); debiendo considerarse estos mismos factores si se plantea la deprescripción (Gnjidic y Le Couteur, 2012).

e) Limitar el uso de carbamazepina en el tiempo en correspondencia a la incidencia de espasmos musculares, y monitorear los niveles de sodio con frecuencia.

f) Modificación de la dieta para incrementar el número de deposiciones.

Una vez analizadas las propuestas de intervenciones con el médico, estas fueron conciliadas con el paciente, para definir el orden de implementación; ya que este tiene derecho de decidir el curso del tratamiento sobre la base de los PS que más le preocupan o perturban.

Al respecto la OMS, en el 2015 planteó que el enfoque de atención debe tener expresamente en cuenta las perspectivas de los individuos, las familias y las comunidades, 
y los considera participantes, así como beneficiarios, de unos sistemas de salud y cuidados a largo plazo que respondan a sus necesidades y preferencias en forma humana e integral.

\section{Evaluación del SFT}

A los dos meses de seguimiento se observó en la paciente evidente mejoría de los síntomas que motivaron la asistencia a consulta; por lo que se consideró una evolución favorable.

Las intervenciones sugeridas y puestas en práctica beneficiaron al paciente, a la familia y a la sociedad, al contribuir a una mejor capacidad intrínseca del paciente y reducir el gasto sanitario.

\section{Conclusiones}

Se hace evidente la necesidad de revisar de manera sistemática la medicación en el adulto mayor, para evitar problemas asociados al uso de medicamentos y contribuir a una mejor calidad de vida.

\section{Referencias Bibliográficas}

American Geriatrics Society. (2019). Updated AGS Beers Criteria ${ }^{\circledR}$ for Potentially Inappropriate Medication Use in Older Adults. J Am Geriatr Soc, 67(4), 674-694. Recuperado de https://www.onlinelibrary.wiley.com/doi/epdf/10.1111/jgs.15767

Cahn, A., Raz, I., Kleinman, Y., Balicer, R., Hoshen, M., Lieberman, N.,..... Cafalu, W. T. (2015). Clinical assessment of individualized glycemic goals in patients with type 2 diabetes: formulation of an algorithm based on a survey among leading worldwide diabetologists. Diabetes Care, 38, 2293-2300.

Cruz, A.J. (2017). Prescripción inapropiada en personas mayores: hora de pasar a la acción. Farm Hosp, 41(1),1-2. doi: 10.7399/fh.2017.41.1.10725.

Delgado, E., Montero, B., Muñóz, M., Vélez, M., Lozano, I., Sánchez, C. y Cruz, A. J. (2015). Mejorando la prescripción de medicamentos en las personas mayores: una nueva edición de los criterios STOPP-START. Rev Esp Geriatr Gerontol, 50(2), 89-96. Recuperado de https://dx.doi.org/10.1016/j.regg.2014.10.005.

Escuela Andaluza de Salud pública. (2017). Revisión de prescripciones para evitar problemas de seguridad. Bol Ter Andal, 32(1), 1-5. http://www.cadime.es/docs/bta/CADIME_BTA_32_1.pdf.

Eschenhagen, T. (2019). Tratamiento de la cardiopatía isquémica. En L.L. Brunton, R. Hilal - Dandan, B.C. Knollmann, (Ed.), Goodman \& Gilman. Las bases farmacológicas de la terapéutica (pp. 489-506). Ciudad de México, México: McGraw-Hill Interamericana.

Eschenhagen, T. (2019). Tratamiento de la hipertensión. En L.L. Brunton, R. Hilal Dandan, B.C. Knollmann, (Ed.), Goodman \& Gilman. Las bases farmacológicas de la terapéutica (pp. 507-526). Ciudad de México, México: McGraw-Hill Interamericana. 


\section{ORANGE JOURNAL}

Gnjidic, D., \& Le Couteur, D.G. (2012). Deprescribing trials: methods to reduce polypharmacy and the impact on prescribing and clinical outcomes. Clin Geriatr Med, 28(2), 237-253.

Krska, J. Howard, R., y Barnet, N. (2017). Deprescribing: tackling increasing polypharmacy. Prescriber, (Jul), 32-36.

Lavan, A. H., Gallagher, P., Parsons, C., y O’Mahony, D. (2017). STOPPFrail (Screening Tool of Older Persons Prescriptions in Frail adults with limited life expectancy): consensus validation. Age and Ageing, 46, 600-607. doi: 10.1093/ageing/afx005.

OMS. (2015). Informe mundial sobre el envejecimiento y la salud. Recuperado de http://apps.who.int/iris/bitstream/10665/186466/1/9789240694873_spa.pdf

Ortega, IL. (2018). Métodos para implementar la atención farmacéutica al adulto mayor, en el contexto de salud cubano (Tesis doctoral). Universidad de la Habana, Ciudad Habana, Cuba.

Poblano, O., Bautista, A.C., Acosta, O., Gómez, P.M. y Saturno, P.J. (2020). Polifarmacia en México: un reto para la calidad en la prescripción. Salud Publica Mex, 62, 859867. https://doi.org/10.21149/11919

Rudolph, J.L, Salow, M.J, Angelini, M.C y McGlinchey, R.E. (2008). The Anticholinergic Risk Scale and anticholinergic adverse effects in older persons. Arch Intern Med, 168, 508-513.

Smith, M.D., Cameron, S., Metcalf, C.S., Karen, S. y Wilcox, K.S. (2019). Farmacoterapia de la epilepsia. En L.L. Brunton, R. Hilal - Dandan, B.C. Knollmann, (Ed.), Goodman \& Gilman. Las bases farmacológicas de la terapéutica (pp. 303-326). Ciudad de México, México: McGraw-Hill Interamericana.

World Health Organization. (2019). Medication safety in polypharmacy: technical report [internet]. Ginebra: WHO, [citado septiembre 22, 2020]. Recuperado de https://apps.who.int/iris/handle/10665/325454. 